\title{
The Incidence of Papillary Thyroid Microcarcinoma in Patients Who underwent Total Thyroidectomy due to Benign Diseases and Our Clinical Approach
}

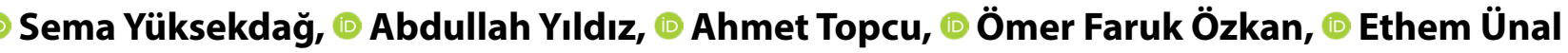

Department of General Surgery, University of Health Sciences Turkey, Umraniye Training and Research Hospital, Istanbul, Turkey

\begin{abstract}
Introduction: To present our experience with incidental papillary thyroid microcarcinoma (PTMC) in patients operated for presumably benign thyroid disease.

Methods: The files of 201 patients operated by the same surgeon for benign thyroid diseases from May 2014 to November 2017 were reviewed retrospectively. All had benign fine needle aspiration cytology and underwent total thyroidectomy. Data regarding demographics, pathology findings, management and outcomes were retrieved.

Results: The mean age of the patients was 46 years (range:17-78) and 159 (79.1\%) of them were female. Twenty-five patients (12.4\%) with incidental PTMC were evaluated. Mean diameter of PTMC was $4.7 \pm 2.4 \mathrm{~mm}$. In 6 patients $(6 / 25,24 \%)$, the tumor was multifocal and in half of these, tumor foci were found in both lobes. In one patient, tumor infiltrated the capsule. All patients received suppressive therapy and referred to the nuclear medicine department for possible radioiodine therapy. One case with capsule infiltration and 3 patients with multifocal tumor (16\%) received radioiodine. Mean follow-up was 2.6 years (range, 4-44 months). All patients were alive and disease-free.

Discussion and Conclusion: PTMC is not an uncommon incidental finding after surgery for presumably benign thyroid disease. The possibility of an underlying PTMC should be taken into account in the management and total thyroidectomy should be considered at least in selected patients.
\end{abstract}

Keywords: Benign thyroid diseases; incidental, total thyroidectomy; microcarcinoma; papillary thyroid carcinoma.

$\mathrm{T}$ he most common malignant tumors of the endocrine system is the thyroid tumor, and papillary thyroid cancers (PTC) constitute more than $80 \%$ of them. The incidence of this type of cancer is increasing due to the use of imaging methods such as ultrasonography (USG), which has been increasingly used in recent years and the use of fine needle aspiration biopsy (FNAB). In general, the ideal treatment for thyroid cancers is total thyroidectomy; if there is involvement of surrounding lymph nodes, it also involves a regional lymph node or a modified radical neck dissection. Considering the size of the tumor, the presence of a multifocal or multicentric focus, and the general condition and age of the patient, radioactive iodine therapy can be applied to patients in the postoperative period. In addition, all patients receive lifelong thyroid stimulating hormone (TSH) suppression therapy. 
All carcinomas of $1 \mathrm{~cm}$ or less in the thyroid gland are called microcarcinomas and the most common type is papillary thyroid microcarcinoma (PTMC). While incidental PTMC can be demonstrated with pathology report in cases operated due to benign thyroid disease, non-incidental form can be diagnosed preoperatively with FNAB and neck USG before surgery. The clinical approach to PTMC varies and a consensus on a common treatment has not been reached yet. Different applications are recommended, from clinical observation, hemithyroidectomy and total thyroidectomy, to total thyroidectomy and central lymph node dissection and postoperative radioactive iodine therapy ${ }^{[1,2]}$. Considering that the lymph node involvement rates reported in PTMC cases are quite low and the 5-year survival rate of PTC is generally above $97 \%$, it is obvious that the clinical course of the disease is good. However, the high frequency of multicentricity and the presence of capsule involvement suggest that a more aggressive treatment may be required and close follow-up may be required in the postoperative period.

Considering the rapid increase in incidental PTMC rates revealed by histopathological examination after thyroidectomies performed for benign thyroid diseases, the importance of established treatment protocols and clinical observations for these patients is evident. In this study, our aim is to share our experience with patients who underwent total thyroidectomy due to benign thyroid disease in our clinic and whose pathology report result was incidentally presented as PTMC.

\section{Materials and Methods}

Ethics Committee approval was obtained from our hospital for the study with the number B.10.1.TKH.4.34.H.GP.0.01/136 with the date 21.11.2018. A total of 201 patients' files, who had total thyroidectomy for a benign thyroid disease (toxic nodular goiter, nodules $4 \mathrm{~cm}$ or larger with benign cytology, etc.) by a single surgeon at the University of Health Sciences, Ümraniye Training and Research Hospital, General Surgery Clinic between May 2014 and November 2017, were analyzed retrospectively.

Before surgery, all patients had benign cytology presented in FNAB and all patients underwent total thyroidectomy in accordance with our clinic's algorithm. Among these patients, those with incidental PTMC in their pathology reports were allocated. Demographic data, histopathology findings, tumor diameter, treatment and results of the patients were evaluated. In addition, patients undergoing radioactive iodine therapy by nuclear medicine department were evaluated. Those diagnosed with preoperative thyroid cancer were excluded from the study.

\section{Results}

While the mean age of the patients was 46 years (range: 17-78), it was found that $159(79.1 \%)$ were female and 42 $(20.8 \%)$ were male. The number of patients who underwent thyroid surgery with a pre-diagnosis of benign thyroid disease and were incidentally diagnosed with PTMC was $25(12.4 \%)$. The mean diameter of PTMC was calculated as $4.7 \pm 2.4 \mathrm{~mm}$.

In six patients $(6 / 25,24 \%)$, the tumor was multifocal, and in half of these, the tumor foci was found in both lobes. There was infiltration of the thyroid capsule in one patient (4\%). Since total thyroidectomy was routinely applied to all benign thyroid diseases with surgery indication in our clinic, no patient in our series required completion thyroidectomy. Intraoperative vocal cord nerve monitoring (NIMResponse ${ }^{\circledast}$ 3.0, Medtronic, USA) was routinely performed in all surgeries. In pathology reports, no involvement was observed in the lymph nodes, which were removed incidentally. Neck dissection was not performed in any of our patients because there was no suspicious finding in thyroid USG performed on all patients during the preoperative period and postoperative neck USG applied to all patients diagnosed with incidental PTMC confirmed the result.

All patients received postoperative suppression therapy (levothyroxine sodium, Abdi Ibrahim Pharmaceuticals, Istanbul, Turkey) and referred to nuclear medicine department for possible adjuvant radioiodine therapy. One case with thyroid capsule infiltration and 3 patients with microtumors in both lobes were detected to undergo radioiodine therapy (16\%). The mean follow-up time was 2.6 years (range 4-44 months). During this time, all of the patients were alive. No tumor recurrence was observed.

\section{Discussion}

Incidental PTMC rates have increased significantly in recent years and as the number of publications on this subject increase, the discussions on the optimal treatment method are also diversifying. While the prevalence of PTMC was reported to be between 1.7 and $35.6 \%$ in autopsy studies ${ }^{[3,4]}$, incidental PTMC rates observed in cases operated for benign thyroid disease ranged from 3 to $17 \%{ }^{[5,6]}$. In our series with a total of 201 patients, who had total thyroidectomy for a benign thyroid disease such as multinodular goiter, toxic nodular goiter, nodules $4 \mathrm{~cm}$ or larger and persistent hyperthyroidism by a single surgeon, that is performed 
routinely within the framework of clinical protocols in our clinic over a period of 3.5 years, we found our incidental PTMC rate as $12.4 \%$, which was consistent with the literature. However, we attribute the considerable amount of data we have found to the total thyroidectomy procedure we prefer to apply due to the rate of recurrence in benign diseases and false negative results that can be seen in FNAB. The routine application of bilateral nerve monitoring in our clinic and the fact that our PTMC rates reach a rate exceeding $12 \%$, enable us to maintain our preference for total thyroidectomy in benign diseases. This method also facilitates the follow-up of all our patients in the postoperative period.

One of the most well-known features of PTC is that the tumor can show high rates of multifocality and bilaterality. Previous studies have reported the multifocality rate of incidental PTMC between 13 and 44\% ${ }^{[7,8]}$. However, recurrence rates are very low (between 0 and $5 \%$ ) in patients with incidental PTMC ${ }^{[8]}$. Considering these rates, it can be said that although the recurrence rate is low, multifocal cases should be followed up more closely. In our series, tumor multifocality was found to be $24 \%$, and half of them had bilaterality. Our results seem to be consistent with the literature.

Although the PTMC recurrence rate is given as $0 \%$ in some old series, it is reported as below $2 \%$ in more recent studies ${ }^{[9,10]}$. However, there are also series that mention rates between 2 and $5 \%^{[11]}$. Since some of these series include non-incidental cases, it was not possible to make a complete distinction. Considering the low rates of lymph node involvement in incidental cases and the low recurrence rates, it can be said that total thyroidectomy alone is an adequate treatment method. However, when faced with incidental PTMC in clinics where unilateral or subtotal thyroidectomy is preferred for benign thyroid disease, considering the multifocality of the tumor, it should be a matter of debate whether supplementary thyroidectomy or close clinical follow-up should be preferred. The preference of total thyroidectomy for also benign pathologies in our clinic strengthens our hand in this regard. Although there was no recurrence $(0 \%)$ in our series, the short mean follow-up period (2.6 years) prevents us from commenting on this issue. Radioiodine therapy seems unnecessary in the postoperative period in incidental PTMC cases. Although there are series in which radioactive iodine is applied, the general opinion is that this treatment method will generally not be required $^{[12]}$. However, considering the multifocality of the tumor and calculating that capsule invasion can be seen, it is important to evaluate all patients in terms of radioiodine therapy, at least on a case-by-case basis. In our series, only $16 \%$ of patients diagnosed with incidental PTMC were treated with radioactive iodine. The indication for these patients was thyroid capsule invasion $(n=1)$ and the presence of the tumor in both lobes $(n=3)$. However, all our patients were evaluated by a nuclear medicine specialist, at least not to take medicolegal responsibility.

\section{Conclusion}

In conclusion, it cannot be said that incidental PTMC is a rare entity in patients operated for benign thyroid disease. The possibility of incidental PTMC should be kept in mind in the treatment of benign thyroid disease and the advantage of total thyroidectomy, at least in selected patient groups, should be considered.

Ethics Committee Approval: Obtained from our hospital for the study with the number B.10.1.TKH.4.34.H.GP.0.01/136 with the date 21.11.2018.

Peer-review: Externally peer-reviewed.

Authorship Contributions: Concept: S.Y.; Design: A.Y., E.U.; Data Collection or Processing: A.T., A.Y.; Analysis or Interpretation: Ö.F.Ö., E.U.; Literature Search: S.Y.; Writing: A.T., S.Y., E.U.

Conflict of Interest: None declared.

Financial Disclosure: The authors declared that this study received no financial support.

\section{References}

1. Noguchi S, Yamashita H, Uchino S, Watanabe S. Papillary microcarcinoma. World J Surg 2008;32:747-53. [CrossRef]

2. Wang TS, Goffredo P, Sosa JA, Roman SA. Papillary thyroid microcarcinoma: an over-treated malignancy? World J Surg 2014;38:2297-303. [CrossRef]

3. Solares CA, Penalonzo MA, Xu M, Orellana E. Occult papillary thyroid carcinoma in postmortem species: prevalence at autopsy. Am J Otolaryngol 2005;26:87-90. [CrossRef]

4. Bondeson L, Ljungberg O. Occult thyroid carcinoma at autopsy in Malmö, Sweden. Cancer 1981;47:319-23. [CrossRef]

5. Bramley MD, Harrison BJ. Papillary microcarcinoma of the thyroid gland. Br J Surg 1996;83:1674-83. [CrossRef]

6. Bron LP, O'Brien CJ. Total thyroidectomy for clinically benign disease of the thyroid gland. Br J Surg 2004;91:569-74. [CrossRef]

7. So YK, Kim MW, Son YI. Multifocality and bilaterality of papillary thyroid microcarcinoma. Clin Exp Otorhinolaryngol 2015;8:174-8. [CrossRef]

8. Gürleyik E, Gurleyik G, Karapolat B, Onsal U. Incidental Papillary Thyroid Microcarcinoma in an Endemic Goiter Area. JThyroid Res 2016;2016:1784397. [CrossRef]

9. Antonaci A, Anello A, Aucello A, Consorti F, Della Rocca C, Giovannone $G$, et al. Microcarcinoma and incidental carcinoma of 
the thyroid in a clinical series: clinical behaviour and surgical management. Clin Ter 2006;157:225-9.

10. John AM, Jacob PM, Oommen R, Nair S, Nair A, Rajaratnam S. Our experience with papillary thyroid microcancer. Indian $\mathrm{J}$ Endocrinol Metab 2014;18:410-3. [CrossRef]

11. Dunki-Jacobs E, Grannan K, McDonough S, Engel AM. Clinically unsuspected papillary microcarcinomas of the thy- roid: a common finding with favorable biology? Am J Surg 2012;203:140-4. [CrossRef]

12. Vlassopoulou V, Vryonidou A, Paschou SA, loannidis D, Koletti A, Klonaris $\mathrm{N}$, et al. No considerable changes in papillary thyroid microcarcinoma characteristics over a 30-year time period. BMC Res Notes 2016;9:252. [CrossRef] 